NUMER. FUNCT. ANAL. AND OPTIMIZ., 11(5\&6), 589-599 (1990)

\title{
ERROR ESTIMATES FOR NONUNIFORM SAMPLING
}

\author{
M. Zwaan
}

Centre for Mathematics and Computer Science

P.O. Box 4079, 1009 AB Amsterdam, The Netherlands.

ABSTRACT

In this paper we consider stability and error estimates for nonuniform sampling. There is a large amount of literature on the subject of error estimates for uniform sampling. In this paper we derive estimates by means of operator norms and obtain new bounds for the amplitude error and the time jitter error, which apply for non uniform sampling.

1980 Mathematics Subject Classification : 46C99, 94A05, 94A15.

Keywords \& phrases: nonuniform sampling, interpolation, moment problem, Hilbert space, Riesz basis, bandlimited function, sinc-function, biorthogonal system, amplitude error, time jitter error, Gram matrix.

\section{INTRODUCTION}

In this paper we consider stability and error estimates for the following problem. Given the data $\left\{g_{i}\right\} \in \ell^{2}(\boldsymbol{I})$ and time markers $\left\{t_{i}\right\}_{i \in I}$, where $g_{i}$ is the value of some function at time $t_{i}$. Here $\boldsymbol{I}$ is an index set which is equal to $\mathbb{Z}$ in the first three sections. In the last section conclusions are stated in the case that $\mathbb{I}$ is a finite subset of $\mathbb{Z}$. The space $\ell^{2}(\mathbb{I})$ is the Hilbert space of sequences of complex numbers $\left\{g_{i}\right\}$ such that

$$
\|g\|_{\ell^{2}}^{2}:=\sum_{i \in I}\left|g_{i}\right|^{2}<\infty
$$

The problem is to find a function $f$ which lies in a Hilbert space of interpolating functions such that

$$
\sqrt{\pi / r} f\left(t_{i} \pi / r\right)=g_{i} \quad \forall i \in \mathbb{I} .
$$

The reason for the factors $\pi / r$ and $\sqrt{\pi / r}$ will become clear in section 1. In this paper we consider stability and error estimates corresponding to problem $(0.1)$.

The first error estimate we compute is the amplitude error, which is defined as follows. Suppose the data $\left\{g_{i}\right\}$ are perturbed to $\left\{g_{i}^{\prime}\right\}$. The solution which corresponds to the perturbed problem is called $f^{\prime}$ and satisfies

$$
\sqrt{\pi / r} f^{\prime}\left(t_{i} \pi / r\right)=g_{i}^{\prime} \quad \forall i \in \mathbb{I} .
$$

A second error estimate is called the time jitter error, which is defined as follows. Suppose the measurement times $\left\{t_{i}\right\}$ are perturbed to $\left\{t_{i}^{\prime}\right\}$. The solution that corresponds to the perturbed problem is again denoted by $f^{\prime}$ and satisfies

$$
\sqrt{\pi / r} f^{\prime}\left(t_{i}^{\prime} \pi / r\right)=g_{i} \quad \forall i \in \mathbb{I} .
$$

The time jitter error is the difference between $f$ and $f^{\prime}$ in the supremum norm, 


$$
e_{\mathrm{tj}}=\left\|f-f^{\prime}\right\|_{\infty}
$$

There is a lot of literature on error estimates in the case of uniform sampling (i.e. $t_{i}=i$, for $i \in \mathbb{Z}$ ). The purpose of this paper is to prove new error bounds which apply for non uniform sampling, by expressing the errors in terms of linear operators and to estimate their norms. In this way a rather transparent derivation of the error bounds is obtained. In the literature alternative methods are being used, which are discussed in the last section.

In the next section we explain some preliminary notions. In section 2 and 3 estimates for the time jitter error and the amplitude error are given. In section 4 conclusions about stability of the problem (0.1) are stated in the case of finite index sets.

\section{PRELIMINARIES}

Most of the material in this section can be found in [8]. The error estimates in this paper are derived by using Riesz bases. Let $\mathcal{H}$ be a separable Hilbert space with inner product $\langle,\rangle_{\mathcal{H}}$ and orthonormal basis $\left\{h_{i}\right\}_{i \in I}$.

Definition 1.1. A system $\left\{\varphi_{i}\right\}_{i \in I}$ is called a Riesz basis for $\mathcal{H}$ if there exists a bounded linear invertible operator $T$ on $\mathbb{P}_{r}$ such that

$$
T \varphi_{i}=h_{i}, \quad i \in \mathbb{I} .
$$

An operator $T$ on a Hilbert space is called invertible if its inverse, denoted by $T^{-1}$, exists and is bounded. Note that in particular any orthonormal basis is a Riesz basis. Throughout this section $\left\{\varphi_{i}\right\}$ denotes a Riesz basis. The Gram matrix $G$ of this system,

$$
G_{i j}:=\left\langle\varphi_{j}, \varphi_{i}\right\rangle_{\mathcal{H}}, \quad i, j \in \mathbb{I}
$$

generates a bounded linear invertible opcrator on $\ell^{2}(\boldsymbol{I})$ (cf. [8], Theorem 9, pp 32-38). Note that $G$ is the matrix representation of $\left(T T^{*}\right)^{-1}$ with respect to the basis $\left\{h_{i}\right\}_{i \in I}$. For, by Definition 1.1

$$
G_{i j}=\left\langle T^{-1} h_{j}, T^{-1} h_{i}\right\rangle_{\mathcal{H}}=\left\langle\left(T T^{*}\right)^{-1} h_{j}, h_{i}\right\rangle_{\mathcal{H}}
$$

Hence

Similarly

$$
\|G\|=\left\|T^{-1}\right\|^{2}
$$

$$
\left\|G^{-1}\right\|=\|T\|^{2}
$$

A system of vectors $\left\{\psi_{i}\right\}_{i \in I}$ which satisfies

$$
\left\langle\varphi_{j}, \psi_{i}\right\rangle_{\mathcal{H}}=\delta_{i j}, \quad i, j \in \mathbb{I}
$$

is called the biorthogonal system of $\left\{\varphi_{i}\right\}_{i \in I}$. Here $\delta$ is the Kronecker delta. In the case that $\left\{\varphi_{i}\right\}$ is a Riesz basis, we have the formula for its biorthogonal system,

$$
\psi_{i}=T^{*} h_{i} .
$$

$T^{*}$ is the adjoint of the operator $T$ of Definition 1.1. So, the Gram matrix $F$ of $\left\{\psi_{i}\right\}_{i \in I}$ is the matrix representation of the operator $T T^{*}$, with respect to $\left\{h_{i}\right\}_{i \in I}$. Any element $f$ lying in $\mathcal{H}$ can be written as (cf. [8] Theorem 5, p. 27)

$$
f=\sum_{j \in I}\left\langle f, \psi_{j}\right\rangle_{\mathcal{H}} \varphi_{j}
$$


In particular, if $f=\psi_{i}$ for $i \in \mathbb{I}$ fixed,

$$
\psi_{i}=\sum_{j \in I}\left\langle\psi_{i}, \psi_{j}\right\rangle_{\mathcal{H}} \varphi_{j}=\sum_{j \in I} \bar{F}_{i j} \varphi_{j}
$$

The bar denotes complex conjugation. Hence,

$$
\psi_{i}=\sum_{j \in I}{\overline{\left(G^{-1}\right.}}_{i j} \varphi_{j}, \quad i \in \mathbb{I} .
$$

An example of a Riesz basis is given in the Hilbert space of bandlimited functions $\mathbb{P}_{r}$. We denote the support of a function $\mathrm{f}$ by $\operatorname{supp}(f)$. The Fourier transform of a function $f \in L^{1}(\mathbb{R}) \cap L^{2}(\mathbb{R})$ is defined by

$$
\widehat{f}(\xi):=\left(\frac{1}{\sqrt{2 \pi}}\right) \int_{R} f(x) e^{-i x \xi} d x .
$$

The Fourier transform can be extended to $L^{2}$-functions and is also denoted by $\hat{f}$.

Definition 1.2. $\quad \mathbb{P}_{r}:=\left\{f \in L^{2}(\mathbb{R}) \mid \operatorname{supp}(\widehat{f}) \subset[-r, r]\right\}$

$\mathbb{P}_{r}$ is a Hilbert space with inner product

$$
\langle f, g\rangle_{P_{r}}:=\int_{R} f(x) \overline{g(x)} d x
$$

and with orthonormal basis, $\left\{h_{i}\right\}_{i \in I}$, where

$$
h_{i}:=\sqrt{r / \pi} \operatorname{sinc}_{r}(\cdot-i \pi / r), \quad i \in \mathbb{I} .
$$

Here the sinc-function is defined by

$$
\operatorname{sinc}_{r}(t):=\left\{\begin{array}{rl}
\frac{\sin (r t)}{r t}, & t \neq 0 \\
1, & t=0
\end{array} .\right.
$$

Let $\left\{t_{i}\right\}_{i \in I}$ be a sequence of real numbers such that

$$
\left|t_{i}-i\right| \leq \alpha<1 / 4, \quad i \in \mathbb{I},
$$

and write

$$
\varphi_{i}=\sqrt{r / \pi} \operatorname{sinc}_{r}\left(\cdot-t_{i} \pi / r\right)
$$

Then, by [8] Theorem 14, pp. $42-44,\left\{\varphi_{i}\right\}_{i \in I}$ is a Riesz basis for $\mathbb{P}_{r}$. Moreover, the operator $T$ of Definition 1.1 satisfies

$$
\|T\| \leq \frac{1}{1-\lambda}, \quad\left\|T^{-1}\right\| \leq 1+\lambda
$$

where

$$
\lambda:=1+\sqrt{2} \sin \pi(\alpha-1 / 4) .
$$

In this case we have that for any $f \in \mathbb{P}_{r}$

$$
(\sqrt{\pi / r}) f\left(t_{i} \pi / r\right)=\left\langle f, \varphi_{i}\right\rangle_{P_{r}} .
$$

We obtain by (1.9) the formula for the Gram matrix $G$

$$
G_{i j}=\operatorname{sinc}_{\pi}\left(t_{i}-t_{j}\right), \quad i, j \in \mathbb{I} .
$$


By (1.7) and (1.2) we estimate the norm of $G^{-1}$,

$$
\left\|G^{-1}\right\|^{1 / 2}=\|T\| \leq \frac{1}{1-\lambda}
$$

where $\lambda$ is given by (1.8). Similarly

$$
\|G\|^{1 / 2}=\left\|T^{-1}\right\| \leq 1+\lambda
$$

It follows from (1.9) that the interpolation problem (0.1) is a special type of moment problem in $\mathbb{P}_{r}$. That is, given a Riesz basis $\left\{\varphi_{i}\right\}_{i \in I}$ with a biorthogonal sequence $\left\{\psi_{i}\right\}_{i \in I}$ and a sequence of complex numbers $\left\{g_{i}\right\}_{i \in I}$ lying in $\ell^{2}(\boldsymbol{I})$, we want to find a function $f$ which lies in $\mathbb{P}_{r}$ such that

$$
\left\langle f, \varphi_{i}\right\rangle_{P_{r}}=g_{i}, \quad i \in \boldsymbol{I} .
$$

The unique solution to this problem is (cf. [8] Example 2, p. 148)

$$
f=\sum_{i \in I} g_{i} \psi_{i}
$$

If $f$ lies in $\mathbb{P}_{r}$, then by the Fourier inversion formula

$$
f(t)=\left(\frac{1}{\sqrt{2 \pi}}\right) \int_{-r}^{r} \hat{f}(\xi) e^{i \xi t} d \xi .
$$

By Jensen's inequality it follows that

$$
|f(t)|^{2} \leq\left(\frac{1}{2 \pi}\right) \int_{-r}^{r}|\widehat{f}(\xi)|^{2} d \xi=\left(\frac{1}{2 \pi}\right) \int_{R}|\widehat{f}(\xi)|^{2} d \xi .
$$

Denoting $\|f\|_{\infty}:=\sup _{t \in R}|f(t)|$, we obtain

$$
\|f\|_{\infty} \leq(1 / \sqrt{2 \pi})\|f\|_{P_{r}}, \quad f \in \mathbb{P}_{r} .
$$

\section{THE AMPLITUDE ERROR}

Let $\left\{g_{i}\right\}_{i \in I}$ and $\left\{g_{i}^{\prime}\right\}_{i \in I}$ be the data corresponding to problem (0.1), and the perturbed data corresponding to $(0.2)$ respectively, both lying in $\ell^{2}(\mathbb{I})$. Throughout this section the index set $\mathbb{I}$ is equal to $\mathbb{Z}$.

The following proposition holds in the case of separable Hilbert spaces $\mathcal{H}$ with orthonormal basis $\left\{h_{i}\right\}_{i \in I}$.

Proposition 2.1 . Let $\left\{\varphi_{i}\right\}_{i \in \boldsymbol{I}}$ be a Riesz basis in a Hilbert space $\mathcal{H}$, with biorthogonal system $\left\{\psi_{i}\right\}_{i \in I}$, and suppose $\left\{g_{i}\right\},\left\{g_{i}^{\prime}\right\} \in \ell^{2}(\boldsymbol{I})$. The following estimate holds,

$$
\left\|\sum_{i \in I}\left(g_{i}-g_{i}^{\prime}\right) \psi_{i}\right\|_{\mathcal{H}} \leq\left\|G^{-1}\right\|^{1 / 2}\left\|g-g^{\prime}\right\|_{\ell^{2}}
$$

The Gram matrix $G$ is given by

$$
G_{i j}:=\left\langle\varphi_{j}, \varphi_{i}\right\rangle_{\mathcal{H}}, \quad i, j \in \mathbb{I} .
$$

\section{Proof:}

By (1.3) and (1.2) we obtain

$$
\left\|\sum_{i \in I}\left(g_{i}-g_{i}^{\prime}\right) \psi_{i}\right\|_{\mathcal{H}}=
$$




$$
\begin{gathered}
\left\|\sum_{i \in I}\left(g_{i}-g_{i}^{\prime}\right) T^{*} h_{i}\right\|_{\mathcal{H}} \leq\|T\|\left\|\sum_{i \in I}\left(g_{i}-g_{i}^{\prime}\right) h_{i}\right\|_{\mathcal{H}}= \\
\|T\|\left\|g-g^{\prime}\right\|_{\ell^{2}}=\left\|G^{-1}\right\|^{1 / 2}\left\|g-g^{\prime}\right\|_{\ell^{2}} .
\end{gathered}
$$

This proves the proposition.

Now take $\mathcal{H}=\mathbb{P}_{r}$ and let $\left\{h_{i}\right\}$ and $\left\{\varphi_{i}\right\}$ be given by (1.5) and (1.6). The biorthogonal system $\left\{\psi_{i}\right\}$ of $\left\{\varphi_{i}\right\}$ is computed by (1.4). The solution to $(0.1)$ is called $f$, the solution to the perturbed problem $(0.2)$ is denoted by $f^{\prime}$, which are $\sum_{i \in I} g_{i} \psi_{i}$ and $\sum_{i \in I} g_{i}^{\prime} \psi_{i}$ respectively (cf. (1.14)). By (1.15) and Proposition 2.1 we obtain,

$$
e_{\text {amp }} \leq(1 / \sqrt{2 \pi})\left\|G^{-1}\right\|^{1 / 2}\left\|g-g^{\prime}\right\|_{\ell^{2}} .
$$

From this estimate it follows that the solution is stable for perturbation of the data, since $G^{-1}$ is a bounded operator on $\ell^{2}(\boldsymbol{I})$. The norm of $G^{-1}$ is estimated by $\left\|G^{-1}\right\|^{1 / 2} \leq \frac{1}{1-\lambda}$, where $\lambda$ is given by (1.8). We see that the norm of $G^{-1}$ in the case of uniform sampling is equal to 1 . In the case of nonuniform sampling the norm of $G^{-1}$ may become larger if $\alpha$ tends to $1 / 4$. The problem (0.1) is called well conditioned if $\left\|G^{-1}\right\|$ is not too large (i.e. close to 1$)$, otherwise it is called ill conditioned. In the case of uniform sampling $(\alpha=0)$ the problem is well conditioned for perturbation of the data and the problem is ill conditioned if $\alpha$ is close to $1 / 4$.

\section{THE TIME JITTER ERROR}

Let $\left\{t_{i}\right\}_{i \in I}$ and $\left\{t_{i}^{\prime}\right\}_{i \in I}$ be the sequences of exact, respectively perturbed time markers. The solution to the exact problem $(0.1)$ is written as $f$ and the solution to the perturbed problem (0.3) as $f^{\prime}$. Define $\varphi_{i}^{\prime}=\sqrt{r / \pi} \operatorname{sinc}_{r}\left(.-t_{i}^{\prime} \pi / r\right)$ and suppose

$$
\left|t_{i}^{\prime}-i\right| \leq \alpha^{\prime}<1 / 4, \quad i \in \mathbb{I} \text {. }
$$

Then, by Section $1,\left\{\varphi_{i}^{\prime}\right\}_{i \in I}$ is a Riesz basis for $\mathbb{P}_{r}$. So, the bounded linear invertible operator $T^{\prime}$ of Definition 1.1, satisfies

$$
T^{\prime} \varphi_{i}^{\prime}=h_{i}, \quad i \in \mathbb{I} .
$$

Moreover (cf formulae (1.11) and (1.12))

$$
\left\|T^{\prime}\right\| \leq \frac{1}{1-\lambda^{\prime}}, \quad\left\|T^{\prime-1}\right\| \leq 1+\lambda^{\prime}
$$

where

$$
\lambda^{\prime}:=1+\sqrt{2} \sin \pi\left(\alpha^{\prime}-1 / 4\right) .
$$

The biorthogonal system of $\left\{\varphi_{i}^{\prime}\right\}$ is denoted as $\left\{\psi_{i}^{\prime}\right\}$, which can be computed by

$$
\psi_{i}^{\prime}=\sum_{j \in I}{\overline{\left(G^{\prime-1}\right)_{i j}}}_{\varphi_{j}^{\prime}}, \quad i \in \boldsymbol{I} .
$$

Here $G^{\prime}$ is the Gram matrix of the system $\left\{\varphi_{i}^{\prime}\right\}$,

$$
G_{i j}^{\prime}=\left\langle\varphi_{j}^{\prime}, \varphi_{i}^{\prime}\right\rangle=\operatorname{sinc} \pi\left(t_{i}^{\prime}-t_{j}^{\prime}\right) .
$$

Again we have a relation between $T^{\prime}$ and $G^{\prime}$,

$$
\left\|G^{\prime-1}\right\|^{1 / 2}=\left\|T^{\prime}\right\|
$$


The solutions $f$ to problem $(0.1)$ and $f^{\prime}$ to problem (0.3) are $f=\sum_{i \in I} g_{i} \psi_{i}$, and $f^{\prime}=$ $\sum_{i \in I} g_{i} \psi_{i}^{\prime}$. The biorthogonal system $\left\{\psi_{i}\right\}$ can be computed by (1.4):

$$
\psi_{i}=\sum_{j \in I} \overline{(G-1)}_{i j} \varphi_{j}
$$

$G$ is given by (1.10) and the system $\left\{\varphi_{i}\right\}$ is given by (1.6).

In order to find an estimate for the time jitter error, we choose the following approach. We look for a perturbation operator $V$, such that $V \varphi_{i}=\varphi_{i}^{\prime}$, for all $i \in \mathbb{I}$. If such an operator exists, then we have the relation $\psi_{i}=V^{*} \psi_{i}^{\prime}$.

The following proposition expresses the difference between $f$ and $f^{\prime}$ in terms of the norm of the operator $T^{\prime}$ from formula (3.1) and $I-V$. This proposition holds for arbitrary separable Hilbert spaces $\mathcal{H}$.

Proposition 3.1. Let $\left\{\varphi_{i}^{\prime}\right\}$ and $\left\{\varphi_{i}\right\}$ be two systems of vectors in a separable Hilbert space $\mathcal{H}$, such that there exists a bounded linear operator $V$ with $V \varphi_{i}=\varphi_{i}^{\prime}$, for all $i \in \boldsymbol{I}$. Let $\left\{\psi_{i}^{\prime}\right\}_{i \in I}$ and $\left\{\psi_{i}\right\}_{i \in I}$ be their respective biorthogonal sequences and assume that $\left\{g_{i}\right\} \in \ell^{2}(\mathbb{I})$. If $\left\{\varphi_{i}^{\prime}\right\}_{i \in I}$ is a Riesz basis for $\mathcal{H}$ (cf. (3.1)), then

$$
\left\|\sum_{i \in I} g_{i} \psi_{i}-\sum_{i \in I} g_{i} \psi_{i}^{\prime}\right\|_{\mathcal{H}} \leq\|I-V\|\left\|T^{\prime}\right\|\|g\|_{\ell^{2}}
$$

Proof:

$$
\begin{gathered}
\left\|\sum_{i \in I} g_{i}\left(\psi_{i}^{\prime}-\psi_{i}\right)\right\|_{\mathcal{H}}=\left\|\sum_{i \in I} g_{i}\left(I-V^{*}\right) \psi_{i}^{\prime}\right\|_{\mathcal{H}} \leq\|I-V\|\left\|\sum_{i \in I} g_{i} \psi_{i}^{\prime}\right\|_{\mathcal{H}}= \\
\|I-V\|\left\|\sum_{i \in I} g_{i} T^{\prime *} h_{i}\right\|_{\mathcal{H}} \leq\|I-V\|\left\|T^{\prime}\right\|\left\|\sum_{i \in I} g_{i} h_{i}\right\|_{\mathcal{H}}=\|I-V\|\left\|T^{\prime}\right\|\|g\|_{\ell^{2}}
\end{gathered}
$$

which proves the estimate.

In the following we prove the existence of this operator $V$, and in addition, we obtain an estimate for the norm of $I-V$ in terms of the difference of $t_{i}$ and $t_{i}^{\prime}$. First we give two Lemma's.

If $\left\{\varphi_{i}\right\}$ is a Riesz basis, then by Definition 1.1, there exists a bounded linear invertible operator $T$ which satisfies

$$
T \varphi_{i}=h_{i}, \quad i \in \mathbb{I} .
$$

Lemma 3.2. Let $\left\{\varphi_{i}\right\}_{i \in I}$ be a Riesz basis for a separable Hilbert space $\mathcal{H}$. Suppose $\left\{\varphi_{i}^{\prime}\right\}_{i \in I}$ satisfies

$$
\sum_{i \in I}\left|\left\langle f, \varphi_{i}-\varphi_{i}^{\prime}\right\rangle_{\mathcal{H}}\right|^{2} \leq C^{2}\|f\|_{\mathcal{H}}^{2}, \quad \forall f \in \mathcal{H}
$$

where $C$ is a constant. Then there exists a bounded linear operator $V$ on $\mathcal{H}$ such that

$$
V \varphi_{i}=\varphi_{i}^{\prime}, \quad i \in \mathbb{I},
$$

and $\|I-V\| \leq\|T\| C$.

Proof:

Let $\left\{\varphi_{i}\right\}$ be a Riesz basis for $\mathcal{H}$, then $T \varphi_{i}=h_{i}$ and $\psi_{i}:=T^{*} h_{i}$ is its biorthogonal system. Define the bounded linear operator $W$ on $\mathcal{H}$ by

$$
W f=\sum_{i \in I}\left\langle f, \varphi_{i}-\varphi_{i}^{\prime}\right\rangle_{\mathcal{H}} \psi_{i}
$$


Then,

$$
\begin{gathered}
\|W f\|^{2}=\left\|\sum_{i \in I}\left\langle f, \varphi_{i}-\varphi_{i}^{\prime}\right\rangle_{\mathcal{H}} \psi_{i}\right\|_{\mathcal{H}}^{2} \leq \\
\|T\|^{2}\left(\sum_{i \in I}\left|\left\langle f, \varphi_{i}-\varphi_{i}^{\prime}\right\rangle_{\mathcal{H}}\right|^{2}\right) \leq\|T\|^{2} C^{2}\|f\|_{\mathcal{H}}^{2} .
\end{gathered}
$$

So $\|W\| \leq C\|T\|$. The adjoint of $W$ is

$$
W^{*} f=\sum_{i \in I}\left\langle f, \psi_{i}\right\rangle_{\mathcal{H}}\left(\varphi_{i}-\varphi_{i}^{\prime}\right)
$$

and

$$
\left(I-W^{*}\right) \varphi_{i}=\varphi_{i}^{\prime}
$$

The result follows by taking $V=I-W^{*}$.

Lemma 3.2 is a slight generalization of Schäfke's Theorem [8], where the system $\left\{\varphi_{i}\right\}_{i \in I}$ is assumed to be an orthonormal basis. The following Lemma is proven in [8] (p. 181, Lemma 3).

Lemma 3.3 . Assume that $\left\{t_{i}\right\}_{i \in I}$ is a sequence of real numbers such that

$$
(\pi / r) \sum_{i \in I}\left|f\left(t_{i} \pi / r\right)\right|^{2} \leq D^{2}\|f\|_{P_{r}}^{2}, \quad \forall f \in \mathbb{P}_{r}
$$

where $D$ is a constant. If $\left\{t_{i}^{\prime}\right\}_{i \in I}$ is a sequence of real numbers which satisfies

$$
\left|t_{i}-t_{i}^{\prime}\right| \leq \gamma, \quad i \in \mathbb{I}
$$

then

$$
(\pi / r) \sum_{i \in I}\left|f\left(t_{i} \pi / r\right)-f\left(t_{i}^{\prime} \pi / r\right)\right|^{2} \leq D^{2}\left(e^{\pi \gamma}-1\right)^{2}\|f\|_{P_{r}}^{2}, \quad \forall f \in \mathbb{P}_{r}
$$

An estimate for the time jitter error can now be derived, by means of a norm estimate for $I-V$. The Gram matrices $G$ and $G^{\prime}$ are given by (1.10) and (3.5), respectively.

Theorem 3.4. Let $\left\{t_{i}\right\}_{i \in I}$ and $\left\{t_{i}^{\prime}\right\}_{i \in I}$ be sequences of real numbers which satisfy,

$$
\begin{aligned}
& \left|t_{i}-i\right| \leq \alpha<1 / 4, \quad i \in \mathbb{I}, \\
& \left|t_{i}^{\prime}-i\right| \leq \alpha^{\prime}<1 / 4, \quad i \in \mathbb{I},
\end{aligned}
$$

and

$$
\left|t_{i}-t_{i}^{\prime}\right| \leq \gamma \leq \alpha+\alpha^{\prime}, \quad i \in \boldsymbol{I} .
$$

The time jitter error can be estimated by

$$
e_{t j} \leq(1 / \sqrt{2 \pi})\left(\left\|G^{\prime-1}\right\|\left\|G^{-1}\right\|\|G\|\right)^{1 / 2}\left(e^{\pi \gamma}-1\right)\|g\|_{\ell^{2}}
$$

\section{Proof:}

If $\left\{t_{i}\right\}$ satisfies the above estimate, then $\left\{\varphi_{i}\right\}$ is a Riesz basis for $\mathbb{P}_{r}$. Let $f \in \mathbb{P}_{r}$,

$$
\begin{gathered}
(\pi / r) \sum_{i \in I}\left|f\left(t_{i} \pi / r\right)\right|^{2}=\left\|\sqrt{\pi / r} \sum f\left(t_{i} \pi / r\right) h_{i}\right\|_{P_{r}}^{2}= \\
\left\|\sqrt{\pi / r} \sum_{i \in \boldsymbol{I}} f\left(t_{i} \pi / r\right) T^{*^{-1}} \psi_{i}\right\|_{P_{r}}^{2} \leq\left\|T^{-1}\right\|^{2}\left\|\sum_{i \in I}\left\langle f, \varphi_{i}\right\rangle \psi_{i}\right\|_{P_{r}}^{2}=\left\|T^{-1}\right\|^{2}\|f\|_{P_{r}}^{2} .
\end{gathered}
$$


So, the conditions of Lemma 3.3 are satisfied with $D=\left\|T^{-1}\right\|$, whence the following inequality is obtained

$$
\begin{gathered}
\sum_{i \in I}\left|\left\langle f, \varphi_{i}-\varphi_{i}^{\prime}\right\rangle_{P_{r}}\right|^{2}=\sum_{i \in I}(\pi / r)\left|f\left(t_{i} \pi / r\right)-f\left(t_{i}^{\prime} \pi / r\right)\right|^{2} \leq \\
\left\|T^{-1}\right\|^{2}\left(e^{\pi \gamma}-1\right)^{2}\|f\|^{2} .
\end{gathered}
$$

Hence the sequences $\left\{\varphi_{i}\right\}$ and $\left\{\varphi_{i}^{\prime}\right\}$ satisfy the conditions of Lemma 3.2, with $C=\left\|T^{-1}\right\|\left(e^{\pi \gamma}-1\right)$. This implies the existence of a linear operator $V$ on $\mathbb{P}_{r}$ such that $V \varphi_{i}=\varphi_{i}^{\prime}$ and

$$
\|I-V\| \leq\|T\|\left\|T^{-1}\right\|\left(e^{\pi \gamma}-1\right) .
$$

Since the $\left\{t_{i}^{\prime}\right\}_{i \in I}$ satisfy (3.8), the system $\left\{\varphi_{i}^{\prime}\right\}_{i \in I}$ is a Riesz basis. By Proposition 3.1 we have that

$$
\left\|f-f^{\prime}\right\| P_{\mathrm{r}} \leq\left(\left\|G^{\prime-1}\right\|\left\|G^{-1}\right\|\|G\|\right)^{1 / 2}\left(e^{\pi \gamma}-1\right)\|g\|_{\ell^{2}}
$$

$f$ and $f^{\prime}$ are solutions to (0.1) and (0.3) respectively. The result follows by (1.15).

A few remarks are in order. From this estimate we see that problem $(0.1)$ is stable for perturbation of the time markers. By the norm estimates (1.11), (1.12) and (3.2) we obtain

and

$$
\begin{gathered}
\|G\|^{1 / 2} \leq 1+\lambda, \\
\left\|G^{-1}\right\|^{1 / 2} \leq \frac{1}{1-\lambda}
\end{gathered}
$$

$$
\left\|G^{\prime-1}\right\|^{1 / 2} \leq \frac{1}{1-\lambda^{\prime}}
$$

Here

$$
\lambda:=1+\sqrt{2} \sin \pi(\alpha-1 / 4)
$$

and

$$
\lambda^{\prime}:=1+\sqrt{2} \sin \pi\left(\alpha^{\prime}-1 / 4\right) .
$$

In the case of uniform sampling ( $\alpha$ is zero and $\alpha^{\prime}$ is close to zero) the problem (0.1) is well conditioned for perturbation of the time markers. If we sampled nonuniformly, especially when $\alpha$ or $\alpha^{\prime}$ is close to $1 / 4$, the problem may become ill conditioned for perturbation of the time markers.

This estimate for the time jitter error can also be obtained by means of the amplitude error $[2,5]$, which is the approach below. Suppose we did measure the data $\left\{g_{i}\right\}_{i \in I}$ at the time markers $\left\{t_{i} \pi / r\right\}_{i \in I}$. Suppose that the sequence of measurement times is registered by our device as $\left\{t_{i}^{\prime} \pi / r\right\}_{i \in \boldsymbol{I}}$. The function we sampled is denoted by $f$, so $g_{i}=\sqrt{\pi / r} f\left(t_{i} \pi / r\right)$. The situation which is registered by our measuring device is false, since it says that the value of $f$ at $t_{i}^{\prime} \pi / r$ is equal to $g_{i}$. However, the true value of $f$ at $t_{i}^{\prime} \pi / r$ is $g_{i}^{\prime}:=(\sqrt{\pi / r}) f\left(t_{i}^{\prime} \pi / r\right)$. So we may consider $\left\{g_{i}^{\prime}\right\}_{i \in I}$ as the exact data and $\left\{g_{i}\right\}_{i \in I}$ as the perturbed data at $\left\{t_{i}^{\prime} \pi / r\right\}_{i \in I}$. With the above notation, we have

$$
f=\sum_{i \in I} g_{i} \psi_{i}=\sum_{i \in I} g_{i}^{\prime} \psi_{i}^{\prime}
$$

and we define $f^{\prime}=\sum_{i \in I} g_{i} \psi_{i}^{\prime}$. The time jitter error is given by

$$
e_{\mathrm{tj}}=\left\|f-f^{\prime}\right\|_{\infty}=\left\|\sum_{i \in I} g_{i} \psi_{i}-\sum_{i \in I} g_{i} \psi_{i}^{\prime}\right\|_{\infty}=\left\|\sum_{i \in I} g_{i}^{\prime} \psi_{i}^{\prime}-\sum_{i \in I} g_{i} \psi_{i}^{\prime}\right\|_{\infty} .
$$


The estimate from Theorem 3.4 can now be derived by formula (2.1) as follows. Let $\left\{t_{i}\right\}$ and $\left\{t_{i}^{\prime}\right\}$ satisfy the conditions of Theorem 3.4. By applying (2.1) with $\psi_{i}^{\prime}$ and $G^{\prime}$ in the role of $\psi_{i}$ and $G$ respectively,

$$
\begin{gathered}
e_{\mathrm{tj}}^{2}=\left\|f-f^{\prime}\right\|_{\infty}^{2} \leq(1 / \sqrt{2 \pi})\left\|f-f^{\prime}\right\|_{P_{r}}^{2}=(1 / \sqrt{2 \pi})\left\|\sum_{i \in I}\left(g_{i}-g_{i}^{\prime}\right) \psi_{i}^{\prime}\right\|_{P_{r}}^{2} \leq \\
(1 / \sqrt{2 \pi})\left\|G^{\prime-1}\right\| \sum_{i \in I}\left|g_{i}-g_{i}^{\prime}\right|^{2}=(1 / \sqrt{2 \pi})\left\|G^{\prime-1}\right\|(\pi / r) \sum_{i \in I}\left|f\left(t_{i} \pi / r\right)-f\left(t_{i}^{\prime} \pi / r\right)\right|^{2} .
\end{gathered}
$$

From the proof of Theorem 3.4, we know that the sequence $\left\{t_{i}\right\}_{i \in I}$ satisfies the condition of Lemma 3.3, with $D=\left\|T^{-1}\right\|$. Hence

$$
e_{\mathrm{tj}} \leq(1 / \sqrt{2 \pi})\left\|G^{\prime-1}\right\|^{1 / 2}\left\|T^{-1}\right\|\left(e^{\pi \gamma}-1\right)\|f\|
$$

The desired estimate now follows by (1.1), (1.3) and (1.14).

This shows that the estimate of Theorem 3.4 can be proven by using (2.1).

\section{CONCLUSIONS AND REMARKS}

In this section we consider the solution of the problem $(0.1)$ in the case that the index set $\mathbb{I}$ is a finite subset of $\mathbb{Z}$ and we state conclusions concerning the amplitude and time jitter error. If the time markers $t_{i}$ are all distinct for all $i \in \mathbb{I}$, then the system $\varphi_{i}=\operatorname{sinc}_{r}\left(.-t_{i} \pi / r\right)$ is linearly independent, so it is a Riesz basis for its linear span.

A solution to problem $(0.1)$ is in this case $f=\sum_{i \in I} g_{i} \psi_{i}$. This solution is not unique, but it is the one with smallest norm among all solutions, the minimal norm solution in $\mathbb{P}_{r}$ to $(0.1)$, see [1]. If both the $t_{i}$ 's and the $t_{i}^{\prime}$ 's are all distinct, then the estimates of Formula (2.1) and Theorem 3.4 are valid. This implies that problem $(0.1)$ in the case of finite index sets is stable for perturbation of the data and the time markers.

If the time markers $t_{i}$ are lying close to each other, then the system $\varphi_{i}$ may become effectively linearly dependent, from a numerical point of view and the matrix $G$ (formula (1.10)) may become singular. Hence the biorthogonal system $\left\{\psi_{i}\right\}_{i \in I}$ which is given by (1.4) cannot be computed. In such a case the algorithm to compute $f$ breaks down. Then the problem (0.1) is ill conditioned for perturbation of the data and of the time markers.

We applied the above interpolation technique in magnetic resonance imaging (MRI), which is a diagnostic method to measure and display cross sections of a human organ, e.g. the beating human heart. A cross section of the heart has to be reconstructed at prescribed measurement times, which are called phases. But since the time markers at which data are measured do, in general, not coincide with the phases, an interpolation technique is used to obtain information at these phases $[9,10]$.

Since there is a vast amount of literature on this subject, we want to make some remarks on error estimates. In the literature the bounds for the time jitter error and the amplitude error are given in the case of uniform sampling.

A bound for the amplitude error is obtained in [4] and [5] by means of the estimate,

$$
\|g\|_{\infty} \leq(\sqrt{r / \pi})\|g\|_{P_{r}}, \quad \text { for } g \in \mathbb{P}_{r} .
$$

It can be proven by the Fourier inversion formula (cf. Section 1) and by the Cauchy-Schwartz inequality,

$$
\begin{gathered}
|g(t)| \leq(1 / \sqrt{2 \pi}) \int_{-r}^{r}|\widehat{g}(\xi)| d \xi \leq \\
(1 / \sqrt{2 \pi})\left(\int_{-r}^{r} 1 d \xi\right)^{1 / 2}\left(\int_{-r}^{r}|\widehat{g}(\xi)|^{2} d \xi\right)^{1 / 2}=(\sqrt{r / \pi})\|g\|_{P_{r}} .
\end{gathered}
$$


Define the amplitude error for uniform sampling by (cf. Section 1)

$$
e_{\text {amp }}:=\left\|f-f^{\prime}\right\|_{\infty}
$$

for $f, f^{\prime} \in \mathbb{P}_{r}$. Note that $f-f^{\prime} \in \mathbb{P}_{r}$. By (4.1) one obtains

$$
e_{\mathrm{amp}} \leq(\sqrt{r / \pi})\left\|f-f^{\prime}\right\|_{P_{r}} .
$$

This estimate from [4] and [5] depends on the bandwidth $r$. If we use (1.15) instead of (4.1), then we would obtain a similar estimate which is independent of $r$,

$$
e_{\mathrm{amp}} \leq(1 / \sqrt{2 \pi})\left\|f-f^{\prime}\right\| P_{\mathrm{r}} .
$$

(4.2) is a special case of (2.1). For, define $g_{i}:=(\sqrt{\pi / r}) f(i \pi / r)$ and $g_{i}^{\prime}:=(\sqrt{\pi / r}) f^{\prime}(i \pi / r)$ for $i \in \mathbb{Z}$. We then have

$$
\left(f-f^{\prime}\right)(t)=\sum_{i \in \mathbb{Z}}\left(g_{i}-g_{i}^{\prime}\right)(\sqrt{r / \pi}) \operatorname{sinc} r(t-i \pi / r),
$$

and

$$
\left\|f-f^{\prime}\right\|_{P_{r}}=\left\|g-g^{\prime}\right\|_{\ell^{2}(\mathbb{Z})}
$$

Hence $(4.2)$ is rewritten as

$$
e_{\mathrm{amp}} \leq(1 / \sqrt{2 \pi})\left\|g-g^{\prime}\right\|_{\ell^{2}(\mathbb{Z})} .
$$

If we take $t_{i}=i$ for all $i \in \mathbb{Z}$ in formula (2.1), then the Gram matrix $G$ (cf. (1.10)) is the identity matrix and (2.1) is the same as (4.3). So, the estimate of [4] and [5] is similar to (2.1) applied for uniform sampling.

Another bound for the amplitude error is derived in [3] Theorem 3.1, via the truncation error (which is not considered here),

$$
e_{\mathrm{amp}} \leq \frac{1+\pi}{\pi}\left(2+r / \pi\|f\|_{L^{1}(R)}\right) \epsilon \ln (1 / \epsilon)
$$

Here $\epsilon:=\sup _{i \in \mathbb{Z}}\left|g_{i}-g_{i}^{\prime}\right|$ and $\|f\|_{L^{1}(R)}:=\int_{R}|f(t)| d t$. This bound holds for all continuous functions $f \in L^{1}(\mathbb{R})$ such that $\operatorname{supp}(\widehat{f}) \subset[-r, r]$.

The time jitter error for stochastic jitter is derived in [5] by applying the mean value theorem to the sampled function. In [2] Theorem 3.11 the time jitter error for uniform sampling (cf. Section 1)

$$
e_{\mathrm{tj}}:=\left\|\sum_{i \in \mathbb{Z}}\left(f(i \pi / r)-f\left(t_{i}^{\prime} \pi / r\right)\right) \operatorname{sinc} r(\cdot-i \pi / r)\right\|_{\infty}
$$

is estimated via the amplitude error and one obtains

$$
e_{\mathrm{tj}} \leq(4 / \beta)\left(\sqrt{5}\|\partial / \partial t f\|_{\infty}+\sqrt{8} M_{f} e^{1 / 4}\right) \delta \ln (1 / \delta) .
$$

Here $t_{i}^{\prime}$ satisfies $\left|\left(i-t_{i}^{\prime}\right) \pi / r\right|<\delta \leq \min \{\pi / r, 1 / \sqrt{e}\}$ for $i \in \mathbb{Z}$ and $f$ has to satisfy (among others)

$$
f(t) \leq M_{f}|t|^{-\beta}, \quad \text { for }|t| \geq 1,
$$

for some $0<\beta \leq 1 . M_{f}$ is a positive constant depending on $f$. 
Applying Theorem 3.4 of this paper to the case of uniform sampling (i.e. $t_{i}=i$ for $i \in \mathbb{Z}$ ) we obtain

$$
e_{\mathrm{tj}} \leq(1 / \sqrt{2 \pi})\left(\frac{1}{\cos \pi \gamma+\sin \pi \gamma}\right)\left(e^{\pi \gamma}-1\right)\|f\|_{P_{r}} .
$$

$t_{i}^{\prime}$ has to satisfy $\left|i-t_{i}^{\prime}\right| \leq \gamma<1 / 4$, for $i \in \mathbb{Z}$. Estimate (4.6) holds for functions in $\mathbb{P}_{r}$, while (4.4) holds for a broader function class. However, in (4.6) the condition (4.5) on the growth of the sampled function $f$ is not needed. $\gamma$ in (4.6) plays the role of $\delta$ in (4.4).

In this paper new bounds for the amplitude and the time jitter error which apply in the case of non uniform sampling, are obtained by using the special structure of the Paley Wiener space (cf. Lemma 3.3) and by expressing the errors in terms of bounded linear operators. Explicit formula's for the error bounds are obtained by estimating the operator norms (cf. (1.11), (1.12) and (3.2)).

ACKNOWLeDGEMENT I gratefully thank Prof. Dr. G.Y. Nieuwland, Dr. J.B.T. M. Roerdink and Dr. H.J.A.M. Heijmans for the great number of comments and their thorough reading of the manuscript.

\section{REFERENCES}

[1] M. Bertero. Regularization Methods for Linear Inverse Problems, In: Inverse Problems. Lecture Notes in Mathematics, no.1225, pp. 52- 113. Springer Verlag, Berlin, 1986.

[2] P.L. Butzer, W. Splettstösser, R.L. Stens. The Sampling Theorem and Linear Prediction in Signal Analysis. Jahresber. Deutsch. Math. Verein, 90 (1988), $1-70$.

[3] P.L. Butzer, W. Splettstösser. On Quantization, Truncation and Jitter Errors in the Sampling Theorem and its Generalizations. Signal Proces., 2 (1980), $101-112$.

[4] A. J. Jerri. The Shannon Sampling Theory - its Various Extensions and Applications, Proceedings of the IEEE. $\underline{65}$ (1966), 1565 - 1596.

[5] A. Papoulis. Error Analysis in Sampling Theory. Proceedings of the IEEE, 54 (1966), no.7. $947-955$.

[6] W. Splettstösser. Unregelmässige Abtastung determinierter und zufälliger Signale. DFG-Schwerpunktporgramm, Digitale Signalverarbeitung, March 1981.

[7] W. Splettstösser. Error Estimates for Sampling Approximation of Non-Bandlimited Functions. Math. Methods Appl. Sci, 1 (1979), $127-137$.

[8] R.M. Young. An Introduction to Nonharmonic Fourier Series. Academic Press, New York, 1980.

[9] M.Zwaan. Dynamic MRI Reconstruction as a Moment Problem. Part II. Riesz bases in $L^{2}$-spaces of vector valued functions. Report AM-R8907, march 1989.

\footnotetext{
Received: November 10, 1989

Revised: March 9, 1990

Accepted: August 3, 1990
} 